\title{
Resolução de problemas abertos como um processo de modelagem didático-científica no Ensino de Física
}

\author{
Solving ill-structured problem as a Didactical-Scientific Modeling process in Physics Education

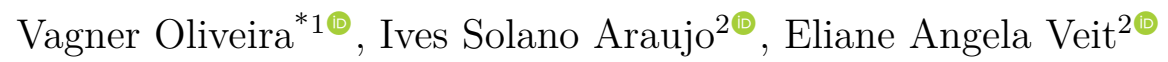 \\ ${ }^{1}$ Instituto Federal de Educação, Ciência e Tecnologia Sul-rio-grandense, Pelotas, RS, Brasil. \\ ${ }^{2}$ Universidade Federal do Rio Grande do Sul, Instituto de Física, Porto Alegre, RS, Brasil.
}

Recebido em 06 de fevereiro de 2020. Revisado em 25 de maio de 2020. Aceito em 29 de junho de 2020.

\begin{abstract}
Pesquisas na área de ensino apontam que se envolver no processo de resolução de problemas abertos têm potencial para a construção de conhecimentos por parte do indivíduo, assim como para o desenvolvimento de um conjunto mais amplo de habilidades, recursos cognitivos, metacognitivos e procedimentais, quando comparados aos problemas que costumeiramente são implementados no contexto do Ensino Médio. Problemas abertos, mais próximos da realidade cotidiana, acadêmica e profissional, deveriam, portanto, ser mais valorizados nas práticas didáticas. No entanto, embora a literatura apresente sugestões e orientações para implementação de tais problemas, as poucas abordagens didáticas com problemas abertos têm indicado que as dificuldades dos estudantes no enfrentamento desses problemas persistem. Associado a isso, não localizamos uma abordagem teórica que considere coerentemente uma teoria de aprendizagem e uma visão epistemológica para a resolução de problemas abertos de física. Frente a esse cenário, o objetivo deste trabalho é apresentar uma articulação teórica entre os elementos centrais do processo de resolução de problemas abertos e a Modelagem Didático-Científica, como forma de auxiliar no desenvolvimento e implementação de estratégias didáticas que auxiliem no enfrentamento de problemas abertos. Palavras-chave: Aprendizagem baseada em problemas, problemas abertos, Ensino de Física, Modelagem didáticocientífica.
\end{abstract}

Physics Education researches indicate that getting involved in the process of solving ill-structured problems has the potential to build knowledge, as well as to develop a broader set of skills, cognitive, metacognitive and procedural resources, when compared to the problems that are usually implemented in the context of high school. Ill-structured problems, closer to everyday, academic and professional reality, should be more valued in didactic practices. However, although the literature presents suggestions and guidelines for the implementation of such problems, the few didactic approaches with ill-structured problems have indicated that students' difficulties in facing these problems persist. Associated with this, we have not found a theoretical approach that consistently considers a learning theory and an epistemological vision for solving ill-structured physics problems. Faced with this scenario, the objective of this work is to present a theoretical articulation between the central elements of the process of solving ill-structured problems and Didactical-Scientific Modeling, as a way to assist in the development and implementation of didactic strategies that help in facing ill-structured problems.

Keywords: Problem-based learning, ill-structured problems, Physics Teaching, Didactical-Scientific Modeling.

\section{Introdução}

A literatura aponta que no ensino de Ciências em geral, e especialmente no ensino de Física, a resolução de problemas é uma das metodologias com maior potencial para favorecer: a construção de conhecimentos específicos, como princípios, leis e teorias [1]; o domínio de procedimentos e técnicas associados à construção do conhecimento científico [2, 3]; o desenvolvimento de habilidades metacognitivas, tais como mecanismos de autocontrole e autorregulação (planejamento, monitoramento e avaliação), que permitem aos estudantes controlar e monitorar sua aprendizagem $[4,5]$; a promoção de melhores crenças

*Endereço de correspondência: vagnerjpn@yahoo.com.br e atitudes dos estudantes relacionadas aos processo de construção do conhecimento [6]; o aperfeiçoamento da estrutura argumentativa e da articulação de raciocínio dos alunos [7].

Um tipo especial de problema, comumente denominado de problema aberte ${ }^{1}$ tem potencial para estimular o desenvolvimento desse amplo conjunto de conhecimentos e habilidades $\$^{2}$ Em particular, são especialmente promissores aqueles que contam com as seguintes características

\footnotetext{
${ }^{1}$ Outros termos também são usados na literatura em inglês para denominar esse tipo de problema, como ill structured problem, openended problem, Fermi problems, real problem e problem estimates [15].

${ }^{2}$ Há problemas abertos abstratos e sem contextualização, porém o foco de nossas atenções está nos problemas abertos definidos neste parágrafo.
} 
definitórias: i) envolvem situações reais, que fazem referência a um evento do mundo cotidiano, acadêmico e/ou profissional .[2, 8]; ii) omitem informações necessárias para a solução [1]; iii) requerem um considerável conjunto de conhecimentos específicos e procedimentais $[3,5]$; iv) possibilitam diferentes caminhos de solução [7]; v) apresentam incerteza em quais conceitos, regras ou princípios são necessários, e como são organizados [9]; vi) inviabilizam a implementação de procedimentos roteirizados para sua resolução [10], e, sendo assim, suas soluções são imprevisíveis; vii) requerem habilidades metacognitivas $[1,4]$.

Sob o ponto de vista do interesse e engajamento do aluno, Jonassen [1] e diversos outros autores [11-13] relatam que o uso de problemas abertos envolvendo questões associadas ao cotidiano do aluno desperta maior interesse do que problemas fechados. Também constatamos maior engajamento emocional e cognitivo dos alunos em um estudo empírico preliminar nosso [14].

Contudo, apesar das potencialidades elencadas, problemas abertos ainda estão pouco presentes no ensino de Física de nível médio [15]; prevalece o uso de atividades de resolução de exercícios de fixação e problemas fechados organizados por tópicos específicos, ao estilo dos problemas presentes em grande parte dos livros didáticos. Esses problemas costumam ser bem definidos, fornecendo todas as informações necessárias para a solução; têm um caminho de solução ideal e esperado; possuem respostas corretas e convergentes (apresentadas ao final do livro ou em gabaritos fornecidos pelo professor). Embora esse tipo de problema possa desempenhar (e desempenha) papel importante na aprendizagem de conceitos físicos, no uso de ferramentas e procedimentos matemáticos [11], propor atividades didáticas somente com problemas fechados falha em mostrar como o raciocínio hipotético-dedutivo pode ser empregado para diminuir a complexidade de problemas reais, tornando-os solucionáveis, e como lidar com incertezas e estimativas. Em particular, a ênfase em problemas exemplares e orientações algorítmicas gerais para soluções de tais problemas não são suficientes para que os estudantes atinjam um bom nível de compreensão das relações entre teoria e realidade [3]. Um cenário de ensino que utilize somente atividades de resolução de exercícios de aplicação e/ou problemas fechados pode estimular os alunos a memorizarem técnicas e procedimentos que possam ser replicados em problemas semelhantes, propostos nas avaliações, como se esse fosse o objetivo principal da educação científica.

Para modificar esse cenário entendemos que é necessário ressignificar as atividades de resolução de problemas de nível médio, a partir da inserção de problemas abertos no ensino de Física. Tendo isso em vista, levantamos a seguinte questão orientadora: Como promover atividades de Ensino de Física no Ensino Médio, relacionadas à resolução de problemas abertos, com ênfase na compreensão de teorias científicas e na construção do conhecimento cientifico?
Embora haja na literatura abordagens alternativas para a implementação de problemas abertos que dão atenção (em diferentes níveis) a aspectos epistemológi$\cos [3,16-21]$, existe uma lacuna no que diz respeito à proposta de um referencial teórico sobre resolução de problemas abertos que considere, de modo simultâneo e coerente, a forma como os estudantes aprendem e aspectos epistemológicos do conhecimento científico [15].

Por outro lado, como será visto na argumentação conduzida ao longo deste artigo, há muito em comum na busca por solução de problemas abertos e na modelagem de situações-problemas da Física. Entenda-se que, grosso modo, modelagem de um sistema ou evento físico (no contexto do ensino) é o processo cognitivo de construção, revisão, exploração e/ou validação, de um modelo (didático-científico) para descrever o sistema ou evento; requer a construção de representações, pois um modelo é uma tentativa de apreensão do real pelo conceitual; requer a utilização de um corpo de conhecimento que extrapola os sentidos; requer intuição e razão; discriminação dos traços mais relevantes; testes de hipóteses, $[22]$.

No caso de modelagem de problemas de Física, dispomos da chamada Modelagem Didático-Científica [23, 24] que engloba elementos relativos à aprendizagem e à construção do conhecimento, pois resulta de uma articulação teórica entre a Teoria dos Campos Conceituais de Vergnaud [25] e as concepções epistemológicas de Bunge [22]. Estudos empíricos embasados nesse referencial mostraram sua pertinência para a formação de professores de nível médio [26] e também para a aprendizagem de Física Geral em nível superior [24].

Neste artigo, por meio de uma articulação teórica entre os principais elementos de abordagens da literatura para a solução de problemas abertos e os da Modelagem Didático-Científica $\left(\mathrm{MDC}^{+}\right)^{3}$ defendemos a tese de que o processo de resolução de problemas abertos de Física pode ser entendido como um processo de mode-

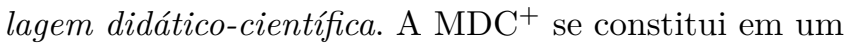
referencial para solução de problemas abertos de Física, com validação empírica [23-24], que considera como os estudantes aprendem e aspectos epistemológicos do conhecimento científico, ou seja, pode preencher a lacuna na literatura citada anteriormente. Adicionalmente, indicamos como delinear atividades de ensino que auxiliem os estudantes a desenvolverem competências associadas à resolução de problemas abertos de Física.

Apesar da articulação teórica proposta no presente artigo não estar acompanhada por resultados de estudos empíricos, previstos como perspectiva futura de investigação, a $\mathrm{MDC}^{+}$, nosso referencial teórico-epistemológico, conta com estudos empíricos que evidenciam suas potencialidades para promover a aprendizagem de Física e do processo de modelagem científica a partir do estudo de situações-problema contextualizadas [14]. Admitindo

\footnotetext{
3 Na seção 3 ficará claro porquê usamos o símbolo ${ }^{+}$na sigla da MDC.
} 
que tais situações se configuram em problemas abertos, passamos a contar com suporte empírico preliminar que nos permite avançar e serve como base para comparações com os resultados de futuros estudos que envolvam atividades de modelagem didático-científico para a resolução de problemas abertos de Física no ensino médio.

É imprescindível enfatizar que neste artigo nossa atenção está voltada para a modelagem de situações-problemas presentes, ou próximas, do mundo vivencial de estudantes de nível médio. As questões a serem propostas aos estudantes focam eventos do mundo real, cujas respostas serão buscadas com base em conhecimentos conceituais e formais de Física. O professor, no entanto, deve estar atento ao fato que os problemas enfrentados na construção do conhecimento científico não necessariamente partem desse tipo de problema. Na medida do possível, em discussões com os estudantes sobre o fazer científico, não pode deixar de: i) enfatizar que não há um único método de se fazer ciência, e que mesmo para a realização de investigações experimentais o processo não é linear; ii) combater o empirismo-indutivismo ingênuo, por exemplo, salientando que as teorias podem se antecipar às evidências experimentais (e.g. a detecção de ondas gravitacionais previstas por Einstein há um século); iii) salientar o aspecto coletivo da construção do conhecimento científico, levando em conta conhecimentos compartilhados com uma comunidade; iv) observar que poderíamos estar interessados em contrastação de diferentes modelos e não começar pela exploração de um problema do mundo real.

Sob o ponto de vista da aprendizagem discente, a solução de problemas abertos via Modelagem DidáticoCientífica é um modo de enfrentar um dos desafios apontados por Vergnaud [27, p. 13]: "um dos problemas do ensino é desenvolver ao mesmo tempo a forma operatória do conhecimento, isto é, o saber-fazer, e a forma predicativa do conhecimento, isto é, saber explicitar os objetos e suas propriedades". O professor ao trabalhar por essa via precisará estar atento aos conhecimentos manifestados pelos estudantes tanto pelo que expressam por escrito e oralmente (forma predicativa), como pelo que fazem (forma operatória) em situação. Inspirados por Vergnaud [25], nossa intenção é que os alunos progridam na conceitualização do real, mais especificamente, de fenômenos físicos, o que depende da compreensão de conceitos, definidos pela tríade: um conjunto de situações que dão sentido ao conceito; um conjunto de invariantes operatórios, em que se baseiam a operacionalidade dos esquemas e; um conjunto de representações simbólicas, que permite representar as propriedades, as situações e os procedimentos de tratamento de um conceito [25]. Cabe salientar que essas e outras premissas da Teoria dos Campos Conceituais (TCC) de Vergnaud estão presentes no referencial da Modelagem Didático-Científica [23, 24], não sendo necessário abordar a TCC de forma separada.

Detalhamos na seção 2 as principais características de problemas abertos de Física que pretendemos abor- dar e os resultados de um estudo teórico [14] que nos permitiu identificar e caracterizar quatros etapas que a literatura aponta para a solução de problemas abertos. $\mathrm{Na}$ seção 3, apresentamos os elementos fundamentais do campo conceitual da Modelagem Didático-Científica e uma discussão sobre suas aplicações para a aprendizagem de Física. Na seção 4, apresentamos a articulação teórica e suas possíveis contribuições para a elaboração de abordagens didáticas que envolvam a resolução de problemas abertos de Física; e na seção 5, os comentários e conclusões. O material complementar contém um exemplo ilustrativo completo, enquanto partes dele são apresentadas ao longo do artigo.

\section{O Processo de Solução de Problemas Abertos de Física}

Ainda que haja consenso dos pesquisadores da área de resolução de problemas sobre a importância do uso de problemas abertos no ensino de Ciências [3, 16, 28-30], nos diferentes níveis da educação, não há acordo sobre a definição a ser adotada e tampouco sobre abordagens e etapas necessárias no processo de solução. Entende-se que, de maneira geral, um problema é descrito em termos de um estado inicial, um estado final desejado, e um conjunto de operações e operadores que permitem transformar o estado inicial no estado final desejado, por meio de uma série de passos e estados intermediários [10], o que não representa um processo linear e não problemático.

Como nos alinhamos com as ideias apresentadas por Jonassen [1, 31], um dos autores mais referenciados em relação a problemas abertos, iniciamos essa seção expondo algumas de suas ideias gerais. Segundo esse autor [1], a resolução de problemas abertos pode ser pensada como um processo de construção, e não como busca sistemática pela solução do problema. É uma sequência de ações na qual o solucionador se envolve em um diálogo com os elementos do problema. Nesse modelo, deve-se reconhecer as perspectivas divergentes, coletar evidências para apoiar ou rejeitar propostas, e sintetizar o próprio entendimento da situação, em vez de buscar uma solução exemplar, automatizada.

Jonassen [31] argumenta que os problemas abertos possuem papel fundamental na idealização de experiências didáticas que facilitem a construção do conhecimento. Tais problemas devem impulsionar a aprendizagem, serem interessantes, relevantes e envolventes, para que os alunos sintam necessidade e motivação para despender esforço e tempo para resolvê-los. Entende, ainda, que propostas de ensino que utilizem resolução de problemas abertos, autênticos, facilitam a construção de conhecimento por parte dos estudantes. O caráter autêntico é atribuído ao envolvimento dos alunos em atividades que apresentem desafios cognitivos do tipo encontrado no mundo cotidiano, que repliquem as estruturas específicas de um contexto. 
Para além das ideias de Jonassen, procuramos levar em conta propostas e resultados de pesquisas de outros autores. Para tanto, baseamo-nos em nossa revisão da literatura sobre problemas abertos de Física [15], entre os anos de 1996 e 2016, que teve os seguintes eixos de análise: 1) perfil da produção acadêmica em resolução de problemas abertos no ensino de Física, no referido período; 2) entendimento da literatura sobre "problemas abertos" e quais os referenciais teóricos adotados nos trabalhos que abordam esses problemas no ensino de Física; 3) metodologias de pesquisa e de ensino associadas com a resolução de problemas; 4) etapas percorridas na resolução de problemas abertos; e 5) recursos cognitivos e procedimentais necessários à resolução desse tipo de problema. Os resultados obtidos nos três primeiros eixos foram apresentados em [15]. Neste artigo avançamos nos dois últimos eixos.

Em consonância com o que já foi apresentado na Introdução, consideramos características gerais dos problemas de nosso interesse as seguintes:

a) correspondem a um ou mais eventos da realidade;

b) despertam interesse na mobilização de tempo e esforço para seu processo de solução;

c) omitem uma ou mais informações relevantes para sua solução;

d) exigem uma análise qualitativa da situação, com o objetivo de identificar os conceitos-chave do problema, as informações relevantes e aquelas que ainda precisam ser encontradas;

e) requerem representação externa dos objetos do mundo real;

f) possibilitam diferentes caminhos viáveis de solução;

g) possuem resultados compatíveis com os do mundo real;

h) abrangem diferentes respostas corretas, dependendo das idealizações realizadas e do grau de precisão desejado;

i) favorecem a construção de um amplo conjunto de conhecimentos conceituais e procedimentais.

j) requerem habilidades metacognitivas.

Para esclarecer o que consideramos exercícios, problemas fechados e problemas abertos, apresentamos três exemplos.

\section{Exemplo de exercício}

Uma partícula varia sua velocidade de $2 \mathrm{~m} / \mathrm{s}$ para 10 $\mathrm{m} / \mathrm{s}$, no decorrer de $4 \mathrm{~s}$. Determine a aceleração média sofrida pela partícula.

\section{Exemplo de problema fechado}

Ao abrir o semáforo de uma grande avenida, um motorista, estudante de Física, pisa no acelerador, imprimindo ao seu automóvel movimento com aceleração constante, até atingir a velocidade de módulo igual a $72 \mathrm{~km} / \mathrm{h}$. Nesse percurso, o automóvel percorre, em linha reta, uma distância igual a $100 \mathrm{~m}$, num intervalo de tempo igual a 10 s. Qual o módulo da aceleração do veículo no trajeto mencionado?

Exemplo de problema aberto
A ilha de Saint Martin, no Caribe, é o destino dos sonhos de muitos turistas que desejam férias em uma praia paradisíaca. A praia de Maho Beach representa um dos pontos turísticos da ilha, em função dos pousos e decolagens do aeroporto Princesa Juliana, cuja pista tem início bem próximo à areia da praia. A atração tornase ainda maior quando as turbinas das aeronaves são acionadas em potência máxima, para iniciar a decolagem, lançando fortes ventos na praia. Alguns turistas, por diversão, arriscam-se posicionando-se atrás da aeronave para sentir as intensas rajadas de vento. Impressionado com as fortes rajadas de vento que as turbinas de um avião comercial são capazes de lançar, um estudante de Física questiona-se sobre o valor da aceleração média de uma aeronave comercial durante um procedimento de decolagem, e decide obter uma estimativa desse valor. Avalie a aceleração média que você supõe que ele possa ter obtido.

Em relação a etapas para solução de problemas abertos, a revisão da literatura mostrou que as estratégias apresentadas para a resolução de problemas abertos podem ser condensadas nos seguintes estágios: 1) definição e representação do problema; 2) elaboração de planos e estratégias de solução; 3) implementação das estratégias de solução; 4) avaliação e monitoramento do processo. Conhecimentos de domínio, conhecimentos procedimentais, habilidades de argumentação e justificação, habilidades metacognitivas, estimativa de grandezas, elaboração e teste de hipóteses, são alguns dos mais relevantes conhecimentos e habilidades necessárias ao enfrentamento dos problemas abertos. Na Figura 1 apresentamos, de maneira esquemática, uma síntese de etapas comuns em abordagens da literatura de problemas abertos $[1,3,8$, $16,29,32]$. A figura representa cada etapa do processo, suas principais características (e respectivos requisitos) e conhecimentos relevantes à solução de problemas abertos de Física [14].

Na primeira etapa - definição e representação - é feita uma análise qualitativa inicial do problema, com o obje-

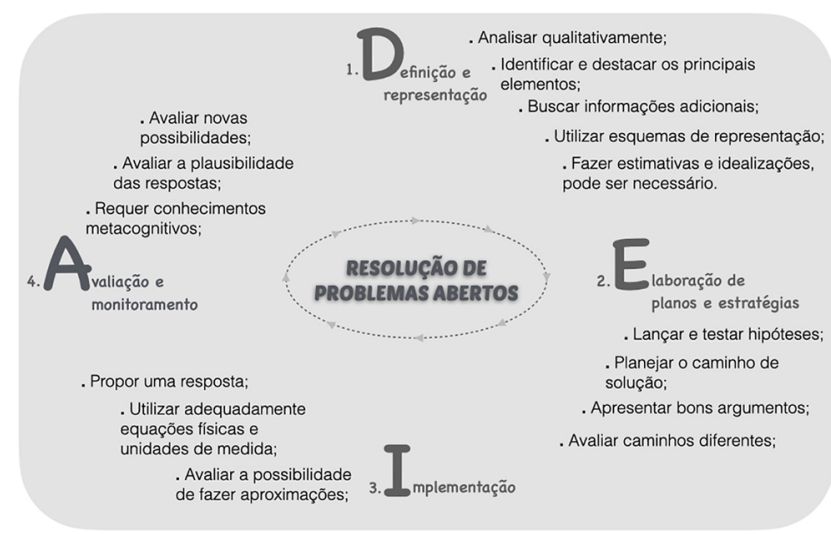

Figura 1: Etapas de resolução de problemas abertos, suas principais características e elementos requeridos para a realização da tarefa [14]. 
tivo de compreender seus aspectos principais, grandezas físicas mais relevantes e relações fundamentais entre elas. É possível que um processo mais profundo de análise e busca de informações ocorra posteriormente para definir melhor o problema. Na sequência, com a finalidade de representar adequadamente a situação problemática, é recomendável que sejam utilizados esquemas, tais como ilustrações pictóricas, gráficos e tabelas. Alguns autores indicam a necessidade de idealizações e realização de estimativas de grandezas físicas. Uma vez realizada a primeira etapa, parte-se à elaboração de planos e estratégias de solução do problema. Esse estágio se dá, em grande medida, pela elaboração e teste de hipóteses, buscando adotar, dentre diferentes possíveis caminhos para a solução, aquele para o qual é possível apresentar os melhores argumentos.

$\mathrm{Na}$ terceira etapa - implementação das estratégias de solução - os estudantes empregam o plano de ação construído na etapa anterior do problema. É nesse estágio que as equações físicas são, de fato, aplicadas, chegando a um resultado final para o problema. A resposta ao problema deverá ser avaliada quanto à coerência com o caminho de solução adotado e com o fenômeno do mundo real. Por fim, a quarta e última etapa - avaliação e monitoramento do processo - consiste em avaliar a plausibilidade do processo como um todo, além de julgar se novos caminhos de solução são úteis para atingir resultados mais condizentes com a realidade.

Embora os trabalhos localizados na literatura tenham optado por descrever o processo de resolução de problemas abertos por meio de etapas, salienta-se que não há, necessariamente, linearidade no processo. Não existem procedimentos que, implementados de forma ordenada, seguindo um passo a passo, possam conduzir, seguramente, a um resultado satisfatório. Entende-se que, em geral, essas etapas estão entrelaçadas, e o processo é repleto de idas e vindas, percorrendo por diversas vezes os estágios, sem que, necessariamente, a ordem apresentada na Figura 1, tanto pela sequência 1, 2, 3 e 4, bem como pelo pontilhado em sentido horário, seja respeitada nas ações do sujeito que resolve problemas abertos.

Em nossa revisão da literatura sobre problemas abertos [15] identificamos em diversos estudos o fornecimento de algum apoio instrucional aos estudantes, a fim de direcionar os esforços do grupo, orientar os procedimentos a serem adotados e/ou auxiliar o desenvolvimento de um raciocínio mais produtivo. Essas assistências à aprendizagem (scaffolding) visam, especialmente, aprimorar em sala de aula o trabalho colaborativo, que possui um papel central nas abordagens socioconstrutivistas de resolução de problemas, e promover atividades reflexivas, permitindo aos estudantes controlar seu próprio processo de aprendizagem [4, 5, 31, 33-35]. São exemplos de assistências à aprendizagem: promoção de interação entre pares
[4], questões-estímuld ${ }^{4}[1,36]$, e iniciadores de sentenças [36].

\section{Modelagem Didático-Científica}

A Modelagem Didático-Científica $\left(\mathrm{MDC}^{+}\right)$[23, 24] foi construída inserindo aspectos do fazer experimental à Modelagem Didático-Científica (MDC) de Brandão, Araujo e Veit ${ }^{5}$ [37]. Em síntese, a modelagem é entendida como um processo por meio do qual criamos, revisamos, validamos e/ou exploramos modelos científicos para reconstruir conceitualmente a realidade através de representações [38].

De acordo com a MDC 6 o campo conceitual da modelagem didático-científica em Física é constituído:

i) pelo conjunto, $S$, de situações que dão sentido aos conceitos associados à noção de modelo e ao processo de modelagem científica em Física [...];

ii) pelo conjunto, I, de invariantes operatórios de caráter geral, associados à noção de modelo $e$ ao processo de modelagem cientifica em Física, e de caráter específico [...];

iii) pelo conjunto, $R$, de representações simbólicas que podem ser usadas para indicar esses invariantes e, consequentemente, representar as situações e os procedimentos de modelagem para lidar com elas [39, p. 4-5].

Invariantes operatórios são os conhecimentos implícitos ou explícitos mobilizados em situação de modelagem que permitem identificar e reconhecer os objetos, suas relações e suas transformações. "Esses invariantes operatórios constituem a base conceitual, implícita ou explícita, que permite selecionar as informações relevantes, definir metas e antecipações, assim como regras de ação adequadas durante o processo de modelagem" [37, p. 355]. Por exemplo, um dos invariantes operatórios primordiais do processo de modelagem associados ao delineamento experimental é Formular questões sobre uma situação física a serem respondidas por meio do uso de modelo(s) científico(s) em uma investigação empírica [23, p. 159].

De maneira esquemática, representamos na Figura 2 etapas do processo de enfrentamento de uma situaçãoproblema de cunho experimental, a ser resolvida por meio da modelagem científica. De acordo com a Teoria dos Campos Conceituais de Vergnaud, o desenvolvimento cognitivo (e a aprendizagem) ocorre a partir da compreensão

\footnotetext{
4 No exemplo discutido na seção 4 e apresentado integralmente no material suplementar, usamos diversas questões-estímulo.

5 Essa, por sua vez, foi estabelecida a partir de uma articulação teórica entre a teoria dos Campos Conceituais de Gérard Vergnaud [25] e as concepções epistemológicas de Mario Bunge [22].

${ }^{6}$ Apresentando a MDC, Vergnaud e Bunge passam a ser referências secundárias. Para compreender com maior profundidade a contribuição desses autores à MDC sugerem-se as referências $[23,24,26$, $37,38]$.
} 


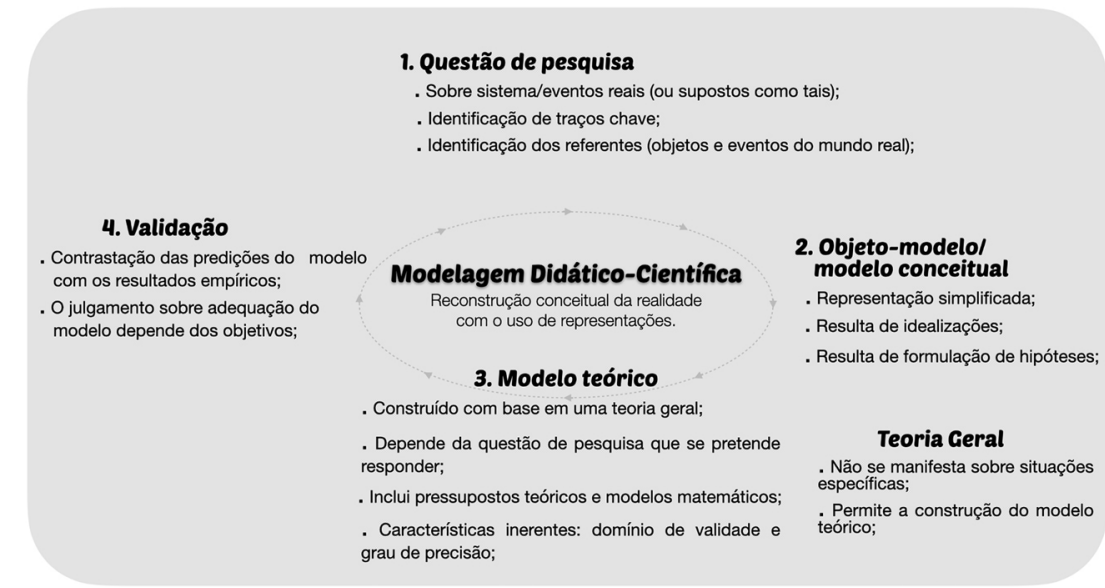

Figura 2: Etapas do processo de Modelagem Didático-Científica $\left(\mathrm{MDC}^{+}\right)$. [Fonte: os autores]

e domínio, progressivo, de diferentes tipos de situações. O conceito de situações pode ser entendido como tarefas, teóricas ou empíricas, que o indivíduo se dispõe a resolver [27], em nosso caso envolvem modelagem de um evento físico. Tudo começa com uma questão de pesquisa. Indivíduos em situação de modelagem formulam questões a respeito de sistemas ou eventos reais (ou supostos como tais) como forma de produção de conhecimento.

A formulação da pergunta requer um tanto de imaginação e de interesse pelo problema em estudo. E aí reside um dos grandes problemas do ensino de Física: com frequência, o professor propõe questões que o aluno não tem o menor interesse em conhecer a resposta. Perguntas que possam ser respondidas pela mera aplicação de uma fórmula, serão respondidas com a mera aplicação da fórmula. Tudo deve começar por uma questão de pesquisa mais geral, cuja resposta não seja meramente do tipo sim ou não, ou um valor numérico. São questões que visam identificar padrões, relevância de certos efeitos. Devem ser elaboradas de tal forma que despertem interesse, de modo que fomentem dedicação de esforços e tempo em seu processo de solução. Nessa etapa se inicia o processo denominado de conceitualização do real, quando, para responder à questão de pesquisa, é preciso definir os objetos do mundo real (referentes: sistemas e eventos reais, ou supostos como tais), seus traços-chave, suas propriedades, relações e transformações. Por exemplo,nessa etapa é necessário se valer de outro invariante-operatório da MDC: Decidir que tipo de representação construir para responder às questões formuladas e Representar a situação física de modo esquemático e à luz de algum campo conceitual específico da Física [23, p. 159].

Em uma segunda etapa, é preciso converter os sistemas/eventos reais em representações conceituais simplificadas, denominadas de modelo conceitual ou objetomodel 7 Nesse processo se percebe o distanciamento entre o sistema real, com toda sua riqueza e complexidade, e o sistema simplificado, que conserva somente

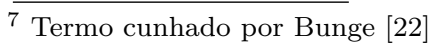

elementos considerados essenciais. Todo modelo é uma representação parcial do sistema real e, como consequência, são características inerentes aos modelos seu grau de precisão e domínio de validade, ambos restritos. Construídos a partir de idealizações e formulação de hipóteses, os objetos-modelo podem ser entendidos como recortes da realidade, constituindo-se em uma representação idealizada, que contém objetos e efeitos ideais. É tão natural trabalhar-se com corpos pontuais, molas sem massas, fios sem resistências, feixes perfeitamente colimados, que muitas vezes os alunos (e mesmo os professores) não se dão conta da necessidade de tomada de decisão na escolha ou construção do modelo conceitual. Que referentes serão considerados? Que fatores poderão ser negligenciados? Um aspecto central é que a construção do objeto-modelo não ocorre no vazio: se dá à luz de uma teoria geral, cuja escolha está condicionada à questão de pesquisa. Essa etapa requer Escolher o modelo científico que amparará o delineamento e a interpretação da investigação empírica.

É na terceira etapa que se constrói o modelo teórico a partir de uma teoria geral. Teorias gerais não se manifestam diretamente sobre situações específicas, contudo por meio da aplicação de leis e princípios de uma teoria geral a uma situação específica, se constrói um modelo teórico, cujas predições podem ser contrastadas com dados experimentais. É comum que modelos físicos sejam confundidos com modelos matemáticos, ou seja, com o conjunto de equações (algébricas, diferenciais ou integrais) que permitem descrever matematicamente a situação física. Porém, é fundamental compreender que modelos físicos não se restringem ao modelo matemático subjacente. Fazem parte do modelo físico o conjunto de pressupostos teóricos (idealizações e hipóteses) concebidos para representar conceitualmente o sistema real.

Na quarta etapa, dá-se a validação do modelo, ou seja, é preciso analisar a razoabilidade dos resultados teóricos preditos pelo modelo contrastando-os com resultados empíricos e/ou outros resultados teóricos assumidos como verdadeiros. Essa contrastação permite avaliar o quão adequado é o modelo para descrever o sistema/evento 
dentro de certo grau de precisão. Claro está que esse julgamento depende das pretensões do modelador. Para determinados propósitos, um desvio de $5 \%$ entre resultados teóricos e experimentais pode ser considerado adequado, em outros casos pode não ser. Se o modelador considerar que o modelo não descreve adequadamente os dados, poderá expandi-lo, incluindo efeitos não considerados na versão inicial do modelo. O refinamento do modelo, de maneira geral, deverá conduzir a uma melhor descrição do sistema/evento em estudo à custa de maior complicação do modelo. Até que ponto vale a pena investir no aprimoramento do modelo é uma decisão do modelador, fortemente associada à questão de pesquisa.

É importante salientar que esse processo de modelagem não representa todo e qualquer problema a ser modelado em Física. Não há um único método pelo qual se faz Ciência. Na Figura 2, assim com discutido na Figura 1, o ordenamento das etapas ilustra uma tendência geral, não seguida à risca. Uma mesma etapa é visitada mais de uma vez e não necessariamente precedida ou seguida pela etapa contígua. Além disso, aqui nos concentramos nos problemas usualmente tratados nos cursos básicos de Física, que visam descrever sistemas e eventos físicos por meio da contrastação empírica, podendo o modelo teórico ser expresso por meio de equações e pressupostos teóricos.

\subsection{Modelagem Didático-Científica $\mathrm{x}$ Modelagem Matemática}

Identificamos em nossa revisão da literatura quatro trabalhos que fazem vinculação entre a solução de problemas abertos de Física e o processo de modelagem. Em [20] e [40], os autores se propõem a mapear os tipos de enunciados e os processos gerados na tentativa de solução dos problemas. Consideram que um dos aspectos fundamentais para atingir uma solução exitosa de um problema é a habilidade de reformulá-lo em termos de sua representação interna, denominando esse processo de "modelo", mas esse tipo de modelo não é o foco do presente trabalho, pois nos preocupamos com representações externas. Ramalho de Souza [41], buscando compreender se o ensino de alguns conceitos de Física poderia ser mediado pela modelagem matemática, conclui que o desenvolvimento exitoso das atividades é viável somente a partir do detalhamento dos conceitos físicos envolvidos na tarefa. Entendemos que a aprendizagem dos conceitos físicos pode seguir pari-passo com a aprendizagem do processo de modelagem. O trabalho de Niss [16], o que mais se aproxima da nossa proposta, apregoa o uso da modelagem matemática, explicitando etapas, procedimentos e conhecimentos que o processo abrange para resolver problemas de Física. No entanto, consideramos que esta seja uma abordagem limitada para auxiliar os estudantes na construção de conhecimentos de Física pelas razões que passamos a argumentar.
Niss [16] defende que o processo de resolução de problemas do mundo real requer a execução de ações e utilização de elementos característicos da modelagem. No entanto, por mais que esse autor $[16,17]$, expresse preocupação com os primeiros estágios de resolução de um problema (e.g. avaliação dos principais elementos e variáveis do problema), o foco de seus estudos está essencialmente na construção do modelo matemático que resolverá o problema. Dos exemplos de resolução de problemas abertos fornecidos [16], percebemos que a análise inicial é muito pragmática, breve e com sucintas discussões - quando ocorrem - a respeito das idealizações, dos referentes, dos principais aspectos do mundo real que serão levados em conta pelo professor na exposição da construção do modelo matemático, dentre outros elementos. A etapa inicial proposta em seu modelo tem como principal objetivo (e parece ser o único) conduzir à construção de um modelo matemático capaz de levar a um resultado adequado para o problema.

Além disso, Niss parece não considerar em seus trabalhos aspectos relacionados à forma como os alunos aprendem e como a ciência é construída. Os conhecimentos físicos são usados, de forma circunstancial, para contextualizar as atividades de modelagem matemática. É dada maior atenção aos procedimentos, processos e etapas do campo da modelagem matemática, do que à construção, por parte do aluno, de conhecimentos específicos de Física. Julgamos que esse possa ser um dos motivos das dificuldades apresentadas pelos estudantes na resolução dos problemas propostos. Niss .[17] argumenta que se os estudantes possuem os conhecimentos do campo específico da Física necessários à resolução dos problemas, poderão aplicá-los aos procedimentos da modelagem matemática; caso contrário, não conseguirão construir o modelo.

Em resumo, o processo de modelagem desenvolvido dessa maneira não enfatiza a compreensão conceitual dos conteúdos de Física. A ênfase está nos aspectos matemáticos. Na perspectiva da modelagem matemática, um determinado conceito é analisado do ponto de vista da Matemática, das funções, equações, estruturas e procedimentos matemáticos, enquanto que para Física são mais relevantes os diferentes contextos e situações. Se o objetivo é alcançar um bom nível de compreensão dos conceitos físicos, segundo as orientações de Vergnaud, a estratégia da modelagem matemática, de focalizar nas estruturas da modelagem e nos procedimentos matemáticos para elaboração de um modelo, pode ser insuficiente.

Além dos trabalhos de Niss, buscamos identificar o discurso teórico da modelagem matemática e a forma como ela é utilizada em atividades de resolução de problemas que envolvem eventos da Física no contexto nacional. Ancorados em uma revisão da literatura sobre modelagem matemática .[42], observamos que um dos principais autores, se não o principal, que propõe atividades de modelagem matemática relacionadas com problemas de Física é Bassanezi [43]. Assim como Niss [16, 17], Bassa- 
nezi [43] também apresenta exemplos de problemas que envolvem eventos físicos resolvidos a partir da perspectiva da modelagem matemática. Na visão do autor, a resolução de um problema sobre um circuito RLC, por exemplo, se concentra na solução das equações diferenciais; a explicitação de idealizações imprescindíveis para se interpretar a realidade à luz de uma teoria é ignorada, assim como a interpretação da solução e discussão do limite de validade do modelo.

O principal argumento que estamos trazendo à discussão é: atividades de modelagem matemática voltadas para o ensino usualmente têm como foco estudar as equações que descrevem o sistema, bem como suas soluções, sendo o conhecimento específico, no caso em análise a Física, apenas um meio para tal fim. Já a modelagem científica, na acepção de Heidemann, Araujo e Veit [24, 44] tem como objetivo principal a compreensão do fenômeno físico e dos conceitos dos campos conceituais da Física e da modelagem envolvidos na tarefa. Fatores epistemológicos da Física importam. Embora contenha equações matemáticas a serem trabalhadas, o modelo físico é mais do que isso. Engloba, entre outras coisas: a delimitação de questões para orientar a investigação dos objetos/eventos reais que farão parte do sistema ou que sobre ele atuarão; avaliação da coerência dos pressupostos teóricos assumidos nas idealizações do sistema frente ao corpo de conhecimentos da Física e resultados empíricos; das representações esquemáticas; e da razoabilidade física das estimativas e aproximações. A solução de um problema aberto de Física via modelagem, requer que sejam discutidas as limitações do modelo, seu grau de precisão, seus possíveis fatores de erros e a plausibilidade das respostas. Apesar de compartilharem um núcleo comum, qual seja, o da representação matemática, a modelagem física e a modelagem matemática, como apresentadas aqui, diferem no foco e na importância dada aos conceitos do conteúdo específico. Se por um lado uma crítica pertinente pode ser feita a uma visão ingênua da matemática meramente como uma linguagem para a Física, da mesma forma, a concepção que a Física se resume apenas a uma aplicação particular da matemática não é adequada.

Reconhecemos que a modelagem matemática é uma das grandes áreas de estudo da Matemática, e que é relevante tanto para sua pesquisa quanto para o seu ensino. No entanto, ressaltamos que, no enfrentamento de problemas que envolvem eventos físicos, o foco da modelagem matemática tem natureza distinta do foco da modelagem física. Tendo isso em vista, sustentamos que nossa proposta de resolução de problemas abertos de Física, vista como um processo de modelagem científica, se afasta dos procedimentos de resolução de problemas realizados pela modelagem matemática.

\section{Articulação teórica entre problemas abertos e modelagem de situações-problema}

A resposta à questão norteadora deste artigo, apresentada na Introdução, foi desenvolvida na tese do primeiro autor deste trabalho [14], onde foi demonstrado que o processo de resolução de problemas abertos de Física pode ser entendido como um processo de modelagem didático-científica, na acepção de Heidemann, Araujo e Veit [24, 44]. Apresentamos, nas seções 4.1 a 4.5, uma síntese dessa construção. Para cada etapa do processo de resolução de problemas abertos, mostradas na Figura 1, argumentamos a favor de uma articulação teórica entre as etapas características do processo de resolução de problemas abertos (seção 2), e os elementos essenciais da modelagem de problemas de Física via $\mathrm{MDC}^{+}$, (seção 3), e, em seguida, apresentamos partes de um problema aberto de Física, concebido a partir de tal articulação teórica. O exemplo completo consta no material suplementar. Motivados pelos resultados da literatura em relação ao uso de assistências à aprendizagem, mencionadas na seção 2, usamos questões-estímulos em todas as etapas do processo de resolução de problemas abertos.

\subsection{Definição e representação do problema}

O processo de resolução começa com a apresentação de um problema aberto, por parte do professor, que pode variar em grau de dificuldade e abstração, desde que mantenha algumas das principais características do tipo de problema de nosso interesse. O problema corresponde a um evento do mundo real, com certo grau de contextualização, com uma questão de pesquisa capaz de instigar os estudantes a mobilizar conhecimentos e esforços para o seu enfrentamento. De acordo com a $\mathrm{MDC}^{+}$, um problema aberto, portanto, deve envolver um conjunto de situações frutíferas, planejadas e fornecidas pelo professor, capazes de desenvolver um repertório de esquemas e representações, permitindo aos estudantes enfrentar problemas e situações cada vez mais complexas.

Para estudar uma situação deve-se, inicialmente, fazer uma análise qualitativa, que tem como objetivo: i) compreender o problema a partir da identificação e explicitação dos conceitos-chave e das relações fundamentais entre as principais grandezas físicas envolvidas; ii) representar os entes do mundo real, ou seja, os referentes do sistema físico apresentado, ou que atuam sobre ele. Tal representação é feita a partir de idealizações, que selecionam os aspectos mais relevantes do problema, frente aos objetivos estabelecidos pela questão orientadora da investigação, desencadeada pela situação física inicialmente apresentada.

\section{Exemplo}

Um pequeno texto introdutório contextualiza o problema que trata do lançamento de uma bola de basquete em direção ao aro. Os estudantes veem um vídeo que 
se popularizou na internet, em que um faxineiro de um ginásio de basquete acerta três arremessos do centro da quadra, após determinar, numericamente, a velocidade com que deveria lançar a bola.

A questão a ser respondida pelos alunos é enunciada da seguinte forma:

Suponha que você deseja reproduzir, com perfeição, a situação apresentada no vídeo: um arremesso perfeito, do centro de uma quadra de basquete, após determinar as grandezas físicas relevantes. Quais são essas grandezas físicas e quais as suas intensidades?

A fim de dar alguma orientação ao aluno, assim como autonomia para que trace o modo em que procederá, seguem algumas questões auxiliares:

a) Vocês compreenderam completamente o problema? Quais as grandezas físicas envolvidas?

b) Quais os conhecimentos que ainda precisam ser obtidos?

c) Qual o tipo de movimento descrito pela bola?

d) Buscando simplificar o evento, quais elementos da realidade vocês irão abordar, e quais idealizações serão assumidas? Por exemplo:

- A massa da bola e o material de que ela é feita deverão ser levados em consideração?

- E a altura do jogador interfere na velocidade e inclinação necessárias para o lançamento perfeito?

- O arremesso ocorrerá em um ginásio fechado ou em uma quadra esportiva a céu aberto? Faz diferença?

e) Para auxiliar na interpretação do problema, vocês podem elaborar uma representação esquemática do evento (um gráfico, um desenho), inserindo todas as informações relevantes ao problema que tem à disposição.

\subsection{Elaboração de planos e estratégias de solução}

As idealizações impostas aos entes envolvidos no sistema físico resultam em um modelo conceitual, que é uma representação simplificada do evento real. Para atingir esse estágio, muitas vezes, é preciso que estimativas de grandezas físicas sejam realizadas. Diversos modelos conceituais podem ser propostos. Quando um modelo conceitual é encaixado em uma teoria geral, se obtém um modelo teórico (i.e. uma teoria específica) como produto. À medida que diferentes idealizações são feitas, ou que cada vez mais elementos do real (ou suposto como tal) são considerados como forma de aperfeiçoamento do modelo, surgem diferentes caminhos para a solução do problema. É importante que o professor auxilie os estudantes nessas tarefas; a fazer conjecturas e antecipações, a esclarecer os objetivos, e reduzir a margem de incertezas. Em outras palavras, o professor "precisa se interessar pelos diferentes componentes do esquema que deseja ver emergir entre os alunos" [45, p. 303].

Exemplo

a) Podemos compreender o movimento da bola como a composição de um movimento horizontal e um movimento vertical? Se sim, quais seriam as características de cada um desses movimentos?

b) Quais estratégias e o caminho de solução vocês pretendem utilizar?

c) Vocês já reconhecem a(s) equação(ões) física(s) que melhor se encaixará(ão) na descrição matemática do problema?

\subsection{Implementação das estratégias}

O desenvolvimento de um modelo teórico em linguagem matemática leva a uma resposta para o problema proposto. No entanto, é possível que o modelo teórico precise passar por uma etapa mais estruturada, de elaboração e teste de hipóteses, que pode envolver operações empíricas. Ao final, as operações empíricas também darão condições de se chegar a uma resposta do problema. $\mathrm{Na}$ $\mathrm{MDC}^{+}$há diversos invariantes operatórios associados a esta etapa, como por exemplo: "Estabelecer procedimentos de controle de variáveis que minimizem a influência dos fatores desprezados no modelo teórico de referência da investigação" [23, p. 159], e "Baseado em hipóteses e/ou no modelo teórico de referência da investigação, avaliar a necessidade de operações empíricas para coleta de dados" [23, p. 160].

\section{Exemplo}

a) Transformem o modelo construído em linguagem matemática, explicitando seus cálculos.

b) Estão utilizando aproximações matemáticas?

c) Vocês veem necessidade de construir algum arranjo experimental para evoluir na resolução do problema?

\subsection{Avaliação e monitoramento do processo}

O resultado obtido deverá, ainda, ser avaliado quanto à sua plausibilidade. Dentre outras coisas, é necessário verificar se o resultado é coerente com o caminho de solução adotado e com a realidade. Caso o resultado não esteja dentro de um espectro aceitável, ou seja, caso as previsões do modelo não tenham atingido o grau de precisão pretendido, novas idealizações podem ser pensadas, reiniciando boa parte do processo de resolução/investigação. Toda essa etapa requer, em grande medida, habilidades metacognitivas, como planejamento e autorregulação. Sob a lente da $\mathrm{MDC}^{+}$, um dos invariantes operatórios a ser manifestado pode ser "Confrontar as predições com as evidências da investigação avaliando se as evidências construídas fornecem um apoio empírico à teoria geral contrastada ou se a discordância entre predições e evidências supera a tolerância aceitável para a investigação" [23, p. 160].

\section{Exemplo}

a) Após chegar a um resultado para o problema, vocês diriam que essa resposta é coerente com a realidade? Não há alguma discrepância entre o resultado, o evento físico, e o caminho de solução adotado?

Vocês podem tentar comparar seus resultados com o vídeo a seguir, no qual o jogador Stephen Curry, do 
Golden State Warriors, no dia 24/02/2017 acerta um arremesso do centro da quadra, em um jogo contra o Los Angeles Clippers.

\section{https://www youtube.com/watch}

b) Vamos agora expandir o domínio de validade do nosso modelo, a partir da inclusão de novos elementos da realidade.

- Considerem que o arremesso está sendo feito em uma quadra aberta, em um dia com ventos consideráveis. O que alteraria no modelo construído?

- O efeito Magnus atua consideravelmente no movimento da bola até a cesta? Se vocês desconhecem esse efeito, podem assistir um vídeo, cujo link está colocado a seguir

\section{https://www youtube.com/watch}

\subsection{Exposição dos resultados e discussões finais}

A exposição dos resultados obtidos e procedimentos adotados no processo de resolução do problema contribui para o desenvolvimento de estruturas argumentativas, construção de conhecimentos específicos e gerais de algum campo conceitual da Física e da modelagem didáticocientífica.

Nessa etapa deve-se estimular os grupos a compararem seus resultados e caminho(s) de solução com outros grupos de estudantes, tendo em vista que o mesmo resultado para um problema aberto pode ser alcançado por caminhos distintos, se valendo de diferentes argumentos. As discussões em grande grupo, resultado desse tipo de atividade, tendem a ser muito enriquecedoras.

É interessante, ainda, que os estudantes sejam solicitados a estabelecer perspectivas para futuros estudos, indicando melhorias que possam ser introduzidas no modelo para que a resposta ao problema apresente melhor grau de precisão, uma nova questão de pesquisa que possa ser analisada como forma de expansão do modelo, ou diferentes situações em que o modelo poderia ser utilizado.

Por fim, como forma de refletir sobre os procedimentos adotados, e aprofundar e consolidar conhecimentos dos estudantes, o professor pode apresentar diferentes possibilidades de criação de modelos conceituais, buscando ampliar o domínio de validade do modelo a partir da inclusão de novos referentes, novas variáveis e parâmetros, e diferentes idealizações.

\section{Exemplo}

Por fim, preparem uma apresentação para o grande grupo, explicitando os resultados obtidos, os procedimentos adotados e os principais argumentos utilizados no processo de resolução do problema.

Tenham em mente que outros grupos também apresentarão suas investigações e propostas de resolução do problema. Preparem-se para avaliar criticamente os outros trabalhos, e fornecer contribuições para aprimorar a resolução dos colegas.
Destacamos que essa construção teórica amplia o número de etapas de resolução de problemas abertos decorrente da revisão da literatura, acrescentando um quinto estágio: exposição dos resultados e discussões finais. Essa nova etapa se inspira no êxito obtido pela metodologia "Episódios de Modelagem" para o enfrentamento de atividades experimentais, via $\mathrm{MDC}^{+}[24]$.

Uma vez alcançada essa etapa final da resolução do problema, na qual os estudantes fornecem uma (ou mais) possibilidade de resposta, fruto de reflexões teóricas e/ou atividades experimentais, é conveniente que sejam propostas atividades para avaliar em que medida as tarefas até então realizadas pelos estudantes fornecem apoio ao modelo teórico adotado. Serão necessárias novas investigações? Existem novos caminhos para resolver o problema? Essa abordagem poderá conduzir os estudantes a um estágio de reflexão sobre os limites do modelo desenvolvido e da resposta obtida. Em síntese, os resultados da análise teórica e/ou experimental podem: i) dar apoio ao modelo teórico adotado; ii) fornecer evidências contrárias a esse modelo; iii) ser descartados, indicando a necessidade de outras operações conceituais ou experimentais.

\section{Exemplo}

a) Após todos os procedimentos já realizados, o grupo entende que os resultados estão de acordo com as previsões do modelo adotado?

b) Embasados nas apresentações e procedimentos adotados pelos colegas, para chegarem a uma resposta para o problema, acreditam que alterando algum elemento do modelo construído por vocês, seriam capazes de atingir resultados com maior grau de precisão? Se sim, qual(is) elemento(s)? E ainda, como pretendem implementar tal modificação?

Entendendo que reflexões teóricas e/ou experimentos científicos suscitam novas questões de pesquisa e evidenciam lacunas no conhecimento existente, o professor pode solicitar que os estudantes estabeleçam perspectivas para futuros estudos, indicando pelo menos uma nova questão de pesquisa que possa ser analisada como forma de expansão do modelo.

O professor também pode apresentar e discutir com os estudantes uma ou mais possibilidades para criação de diferentes modelos, conceituais e teóricos, além de avaliação empírica. Isso se justifica na medida em que a apresentação e discussão, por parte de um especialista, dos critérios e procedimentos assumidos na construção de um modelo teórico, tende a contribuir para o aprofundamento de conhecimentos e reflexão dos estudantes em relação às suas investigações.

Para a avaliação dos estudantes no enfrentamento de problemas abertos, o uso de um protocolo contendo itens relevantes para cada etapa do processo de solução pode ser particularmente útil. Por exemplo, para a etapa de definição e representação do problema, alguns dos itens a serem avaliados podem ser a identificação do tópico do conteúdo, considerando relações fundamentais entre as grandezas físicas do problema; identificação das informa- 
ções relevantes, destacando o que precisa ser conhecido; e representação dos referentes do sistema físico ou que sobre ele atuam. Já na etapa de elaboração de planos e estratégias de solução itens como a realização de estimativas com valores típicos do mundo real; apresentação de bons argumentos para a solução; planejamento do caminho de solução; e a inclusão de diferentes referentes no modelo adotado podem ser considerados. Na etapa de implementação das estratégias por sua vez podem ser levados em conta a adoção de um caminho de solução coerente; explicitação das equações físicas adequadas para o enfrentamento do problema; utilização correta das unidades das grandezas físicas relacionadas; e elaboração e teste hipóteses. Na etapa seguinte, a avaliação e monitoramento do processo podem ser avaliadas a plausibilidade das respostas, em função do caminho de solução adotado e da realidade (grau de precisão); e a inclusão de novos elementos da realidade, a fim de expandir o domínio de validade do modelo. Por fim, na etapa de exposição dos resultados e discussões finais podem ser consideradas a exposição dos argumentos, e tomadas de decisão do grupo no decorrer do processo; a comparação e discussão das soluções com diferentes grupos; e a participação crítica nas discussões finais. A exemplificação dos itens de um possível protocolo, aqui apresentados, podem servir de base para o planejamento docente da avaliação da resolução de problemas abertos, mas de modo algum restringem as possibilidades de acréscimo ou supressão de pontos a serem considerados. Além disso, para cada item, será necessário estabelecer uma escala que indique o quanto as respostas apresentadas se aproximam ou se afastam dos resultados desejados pelo professor, o que pode variar sensivelmente dependendo do contexto educacional e dos objetivos de ensino estabelecidos. Cabe também destacar as possibilidades de uma avaliação setorial, envolvendo os itens associados a cada etapa, e de uma avaliação global do processo de resolução de problemas abertos.

A fim de ilustrar em maior detalhe como a articulação teórica pode orientar a construção de uma abordagem didática para resolução de problemas abertos, apresentamos, no material suplementar, como dito anteriormente, a versão completa do problema aberto usado como exemplo nesta seção. No entanto, cabem ainda algumas observações a respeito da construção desse e de outros problemas a serem tratados em nossa abordagem.

Inicialmente, salientamos que não há a necessidade de trabalhar todas as etapas do processo de resolução com a mesma ênfase em cada problema abordado. Por exemplo, o professor pode escolher enfatizar a primeira etapa ao trabalhar os primeiros problemas abertos com seus estudantes, e discutir brevemente como proceder com as etapas seguintes, mas tomando sempre o cuidado de apresentar o processo completo. Assim, pode-se gradualmente avançar no ganho de compreensão do processo de resolução, conforme mais problemas vão sendo discutidos e maior entendimento se vai conquistando, mas sem perder a visão geral do processo. Cabe salientar que a busca deve ser por oportunizar o desenvolvimento das habilidades dos estudantes em resolver problemas abertos e não que prontamente sejam capazes de reproduzir soluções completas e em profundidade.

O objetivo principal da resolução de problemas abertos via modelagem didático-científica é favorecer a construção do conhecimento científico por parte dos estudantes a partir de atividades que integrem conhecimentos específicos de um dos campos conceituais da Física (e.g. conceitos introdutórios à Dinâmica) com conhecimentos do campo conceitual da modelagem didático-científica (e.g., conceito de modelo, idealização). Não se trata de primeiro aprender os conceitos da Física para depois aprender modelagem, mas sim progredir de forma conjunta - e gradualmente - nos dois campos.

$\mathrm{Na}$ instrução formal tradicional, os conhecimentos costumam ser ensinados a partir da fragmentação do conhecimento global que se pretende introduzir aos estudantes, em elementos menores, para que, progressiva e linearmente, sejam ensinados. Espera-se que somente ao final do processo o estudante desenvolva uma compreensão mais holística do que foi estudado. Como consequência, as abordagens didáticas empregadas se caracterizam por começar por casos particulares, que cobrem uma pequena parte de algo mais amplo, que somente ao final de um tempo considerável o estudante terá condições de compreender. Entretanto, quando um conceito é trabalhado em sala de aula de maneira isolada, com situações que apresentam sentido restrito aos muros escolares, a tendência é que os estudantes não atribuam significado ao conceito, além de se desmotivarem.

Em nosso enfoque, partimos de outro ponto de vista. Entendemos que seja possível construir com os estudantes uma noção mais ampla do conhecimento em um nível mais geral e, gradativamente, aprofundamos o nível do conhecimento trabalhado sem perder a noção do todo. $\mathrm{Na}$ perspectiva da nossa articulação teórica, significa dizer que, em cada atividade didática de resolução de problemas abertos, mais de um conceito, tanto do campo conceitual da modelagem didático-científica quanto do campo específico da Física serão trabalhados. Por exemplo, sob o ponto de vista da modelagem, em todas atividades, por mais simples e introdutórias que possam ser, os estudantes serão estimulados a construir uma representação simplificada da realidade, elaborando um modelo conceitual, a partir de idealizações, e chegarão a alguma solução preliminar do problema, a partir da adoção de um modelo teórico, que poderá vir a ser revisado e melhorado oportunamente. Dessa forma, mesmo nas atividades iniciais, em que naturalmente poucos conhecimentos costumam ser apresentados pelos estudantes, uma visão ainda que superficial do processo completo de resolução de problemas via modelagem didático-científica deverá ser desenvolvida.

Nosso foco neste artigo é no ensino de Física em nível médio, porém a articulação teórica apresentada nas seções 4.1 a 4.5 é aplicável para a proposição de proble- 
mas abertos de Física, a serem enfrentados por via da modelagem didático-científica, também no nível superior. Obviamente o grau de aprofundamento nos conceitos da modelagem e, por consequência, o domínio requerido dos alunos no uso dos invariantes-operatórios exemplificados nas seções 4.1 a 4.5 , é bastante distinto quer se trabalhe no nível médio, quer no nível superior. Mesmo no nível superior, poderá haver uma diversidade no nível de aprofundamento quer se trate de cursos de bacharelado e licenciatura em Física, quer se trate das disciplinas de Física Geral em outros cursos, como Engenharia ou Biologia.

\section{Considerações Finais}

Neste trabalho, argumentamos a favor da ressignificação das atividades de resolução de problemas de Física, no ensino médio, a partir da inserção de problemas abertos, que possibilitam aos estudantes desenvolverem um conjunto de conhecimentos e habilidades mais amplo, quando comparados aos problemas e exercícios tradicionalmente implementados nas abordagens didáticas usuais.

Dispostos à adoção dos problemas abertos, lançamos a seguinte questão orientadora: Como promover atividades de Ensino de Física no Ensino Médio, relacionadas à resolução de problemas abertos, com ênfase na compreensão de teorias científicas e na construção do conhecimento científico? A resposta construída ao longo desse trabalho é: pela via da modelagem-didático-científica [23, 24]. Nas seções 4.1 a 4.5 mostramos como etapas comuns de abordagens da literatura para problemas abertos, enriquecidas com assistências à aprendizagem, podem ser compatibilizadas com a $\mathrm{MDC}^{+}$, referencial teórico-metodológico para a condução de atividades de modelagem científica de problemas de Física. As ações propostas nessas seções orientam a construção, implementação e enfrentamento de problemas abertos. Espera-se que o uso de tais orientações na concepção e implementação de atividades possam auxiliar os estudantes a desenvolverem competências associadas ao processo de resolução de problemas abertos de Física.

Um dos pontos centrais de nossa proposta é o que denominamos de abordagem global, ou seja, os estudantes começam a trabalhar com a modelagem de problemas de física em um nível mais geral e superficial, enfatizando a necessidade de responder à questão por meio de uma representação simplificada que leve a alguma resposta tentativa, não única, à questão inicial; gradativamente o nível do conhecimento requer maiores exigências em cada etapa do processo de solução, tanto em termos de invariantes operatórios de caráter geral, associados à noção de modelo e ao processo de modelagem científica em Física, quanto os de caráter específico, associados à matéria de estudo. Como discutido na seção 3, a compreensão de que modelagem é um campo conceitual subjacente aos campos conceituais da Física, constituído pelo conjunto de situações de física que dão sentido a conceitos relevantes para o processo de modelagem, requer que diversas situações sejam tratadas, porém não necessariamente será dada igual ênfase a todos os conceitos envolvidos. Por ex., nos estudos de Heidemann, Araujo e Veit [24], foram trabalhadas com estudantes de graduação de Física (Licenciatura e Bacharelado), quatro situações-problemas referentes a eventos físicos, por meio da modelagem didático-científica, com diferentes focos: no estudo de pêndulos foi dada ênfase aos conceitos de domínio de validade, grau de precisão, idealização, aproximação, evidência e predição, enquanto no estudo de sistema de amortecimento automotivo, a ênfase foi em controle de variáveis, experimento, delineamento experimental e contrastação empírica. Em se tratando de graduandos de Física, é plenamente justificável que todos esses conceitos, e outros, de modelagem sejam vistos com algum grau de profundidade. No caso de ensino médio, pretende-se com a abordagem global que os estudantes construam gradativamente noções sobre processo inteiro de modelagem, por meio de sucessivas atividades de solução de problemas abertos, trabalhando conhecimentos predicativos e operatórios, mas com a formalização adequada ao nível médio.

Outro ponto fundamental é que os estudantes, ao tomarem conhecimento do problema a ser resolvido, sintam-se estimulados a mobilizar esforços e conhecimentos para enfrentá-lo, encarando-o como um problema autêntico, com resultado possivelmente útil para sua vida cotidiana, acadêmica ou profissional. Nesse sentido, é crucial que os problemas abertos sejam contextualizados, o que pode ser feito a partir de textos escritos, imagens e/ou vídeos, e também problematizados, ou seja, devem ser propostas questões instigantes que façam sentido para o estudante e que ele se engaje cognitiva e emocionalmente.

Em nossa proposta, a última etapa de atividades envolvendo solução de problemas abertos consiste na apresentação das soluções dos grupos para o grande grupo e uma discussão final. A premissa básica que sustenta essa etapa, respaldada por resultados da literatura [32, $33,46]$ é que a exposição de conceitos ou de ideias pode contribuir para o desenvolvimento de estruturas argumentativas e solidificação de conhecimentos. Ao requerer que os estudantes compartilhem com o grande grupo os procedimentos e raciocínios adotados na solução do problema, assim como as suas conclusões, o professor torna o aluno ativo no aprendizado, enquanto assume o papel de mediador das discussões, devendo garantir: i) que haja um ambiente colaborativo em sala de aula, de tal forma que os estudantes se sintam motivados e com liberdade de expressar suas ideias; ii) certa qualidade nas discussões, introduzindo questionamentos e termos técnicos à medida do necessário; iii) a promoção de debates explícitos a respeito do fazer científico, para que as crenças do estudantes evoluam no sentido de crenças científicas sobre a natureza da Ciência [47].

O desenvolvimento de habilidades metacognitivas é um dos aspectos mais enriquecedores de atividades com 
problemas abertos. Tais habilidades são requeridas em praticamente todas as etapas da resolução de problemas abertos, desde a reflexão sobre os conhecimentos que precisam ser adquiridos para a execução da tarefa até o ajuste de caminhos de solução, análise da plausibilidade de uma resposta, novas possibilidades de solução, etc. Analisar a razoabilidade de um modelo exige habilidades metacognitivas, como a autorregulação, que permite aos estudantes tomarem consciência do transcorrer de suas atividades, de suas ações na tarefa, permitindo controlar e ajustar suas condutas [48]. Além disso, os problemas abertos que tratamos, por corresponderem a um evento do mundo real, por necessitarem de uma construção teórica e racional para a sua resolução, tendem a melhorar as crenças epistemológicas dos estudantes, possibilitando melhor entendimento da relação entre teoria e realidade $[6,21,49]$. Para instigar as habilidades metacognitivas e induzir alguns procedimentos a serem adotados, recomendamos fortemente o uso de assistências à aprendizagem na forma de questões-estímulos.

O tempo requerido para o desenvolvimento de atividades com problemas abertos é uma séria dificuldade a ser enfrentada. De maneira geral, é necessário mais tempo para que problemas abertos sejam resolvidos pelos estudantes do que problemas/exercícios tradicionais. Uma das razões para isso é que, além de poder propiciar aprendizagem de conteúdos específicos da matéria de estudo, as atividades de enfrentamento de problemas abertos podem auxiliar no desenvolvimento de habilidades cada vez mais necessárias para a formação do aluno como cidadão, tais como seleção de informações, tomada de decisão, pensamento crítico, autonomia e autorregulação. Uma possibilidade de ampliar o tempo destinado para atividades de resolução de problemas abertos está no desenvolvimento de projetos em iniciativas de ensino não-formais, como Feiras de Ciências, cuja preparação possa ser feita em momentos que vão além dos períodos destinados à disciplina de Física.

É oportuno observar que a abordagem de problemas abertos aqui proposta pode ser vista como um caso particular de Aprendizagem Baseada em Projetos (ABP). A APB abarca uma grande variedade de alternativas [50]. Há desde propostas que implicam reforma curricular, como a de Hernandez e Ventura [51], até alternativas que podem ser aplicadas em algumas poucas aulas, como a de Bender [52]. Segundo Bender [52, p. 15],

\section{"[...] a ABP pode ser definida pela utilização de projetos autênticos e realistas, baseados em uma questão, tarefa, ou problema alta- mente motivador e envolvente, para ensinar conteúdos acadêmicos aos alunos no contexto do trabalho cooperativo para a resolução de problemas."}

De maneira geral, os projetos propostos por Bender envolvem problemas abertos mais amplos que os considerados em nossos estudos, muitas vezes interdisciplinares e que requerem várias aulas para o seu desenvolvimento. Todavia, não estão excluídos problemas mais restritos e nesse sentido se poderia dizer que a nossa proposta é um caso particular da ABP. Duas são as principais diferenças entre essas duas abordagens: i) o tempo de envolvimento na solução do problema; enquanto em nosso caso o processo de solução está limitado a alguns períodos de aula, um problema típico da ABP costuma envolver iniciativas de médio prazo (um ou dois meses de aula, na ABP de Bender), e longo prazo (um ano ou um curso, na APB de Hernandez e Ventura); ii) a materialidade da resposta dos estudantes à atividade proposta; enquanto em nosso caso a resposta se dá com a apresentação oral e/ou escrita dos argumentos e tomadas de decisão adotadas, na ABP prevê-se a produção de algum produto/artefato.

Como perspectiva de continuação do trabalho destacamos a necessidade de ampliação do número de atividades de resolução de problemas abertos a serem trabalhadas no Ensino Médio, a realização de estudos em sala de aula que permitam avaliar empiricamente a articulação teórica apresentada neste artigo e como planejar atividades em projetos maiores a partir da articulação teórica desenvolvida nesse trabalho.

\section{Agradecimentos}

Agradecemos aos pareceristas, cujas críticas e sugestões em muito contribuíram para a melhoria do trabalho. Ives Solano Araujo agradece ao apoio recebido pelo $\mathrm{CNPq}$ na forma de Bolsa Produtividade nível 2.

\section{Material Suplementar}

O seguinte material suplementar está disponível online: Apêndice.

\section{Referências}

[1] D.H. Jonassen, Educ. Technol. Res. Dev. 45, 65 (1997).

[2] D.H. Jonassen, Learning to Solve Problems: an instructional design guide (John Wiley \& Sons, Nova Jersey, 2004).

[3] L. Clement e E.A. Terrazzan, Exp.Ens. Ciênc. 7, 98 (2012).

[4] X. Ge e S.M. Land, Educ. Technol. Res. Dev. 51, 21 (2003)

[5] X. Ge e S.M. Land, Educ. Technol. Res. Dev. 52, 5 (2004).

[6] C.A. Ogilvie, Phys. Rev. Spec. Top. - Phys. Educ. Res. 5, 1 (2009).

[7] N. Shin, D.H. Jonassen, e S. McGee, J. Res. Sci. Teach. 40, 1 (2003).

[8] D. Fortus, Sci. Educ., 93, 86 (2009).

[9] D. Jonassen, J. Res. Technol. Educ. 35, 36 (2003).

[10] A.D.T. Gomes, A.T. Borges e R. Justi, Investig. Ens. Ciênc., 13, 187 (2008).

[11] L.O.Q. Peduzzi, Cad. Cat. En. Fís. 22, 229 (1997). 
[12] M. Enghag, P. Gustafsson e G. Jonsson, Res. Sci. Educ. 37, 449 (2007).

[13] M. Enghag, P. Gustafsson e G. Jonsson, Int. J. Sci. Math. Educ. 7, 455 (2008).

[14] V. Oliveira, Resolução de problemas abertos para aprendizagem de Física no Ensino Médio na perspectiva da Modelagem Didático-Científica. Tese de Doutorado, Universidade Federal do Rio Grande do Sul, Rio Grande do Sul (2018).

[15] V. Oliveira, I.S. Araujo, e E.A. Veit, Rev. Bras. Ens. Fís. 39, 1 (2017).

[16] M. Niss, Lat. - Am. J. Phys. Educ.6, 3 (2012).

[17] M. Niss, Int. J. Sci. Math. Educ. 15, 1441 (2017).

[18] B.M. Bravo e M.A. Pesa, Investig. Ens. Ciênc. 10, 337 (2005).

[19] S. Hunsche e D. Auler, Rev. Electr. Ens. Cienc. 11, 1 (2012).

[20] M.H. Truyol e Z. Gangoso, Investig. Ens. Ciênc. 15, 463 (2010).

[21] N. Pizzolato, C. Fazio, R.M. Sperandeo Mineo e D. Persano Adorno, Phys. Rev. Spec. Top. - Phys. Educ. Res. 10, 010107 (2014).

[22] M. Bunge, Teoria e realidade (Editora Perspectiva, São Paulo, 1974).

[23] L.A. Heidemann, Ressignificação das atividades experimentais no ensino de física por meio do enfoque no processo de modelagem cientifica. Tese de Doutorado, Universidade Federal do Rio Grande do Sul, Rio Grande do Sul (2015).

[24] L.A. Heidemann, I.S. Araujo e E.A. Veit, Investig. Ens. Ciênc. 23, 352 (2018).

[25] G. Vergnaud, em ANAIS 10 Seminário Internacional de Educação Matemática do Rio de Janeiro (Rio de Janeiro, 1993).

[26] R.V. Brandão, I.S. Araujo e E.A. Veit, Alex. Rev. Educ. Ciênc. Tec. 12, 85 (2019).

[27] G. Vergnaud, Rev. GEMPA 17, 287 (1996).

[28] L. Clement e E. Terrazzan, Rev. Electr. Investig. Educ. Cienc. 6, 87 (2011).

[29] V. Shekoyan e E. Etkina, AIP Conf. Proc., 951, 192 (2007).

[30] M. Barkovich e A. Carreño, Lat. Am. J. Phys. Educ. 7, 63 (2013).

[31] D. Jonassen, ETR\&D 45, 65 (1997).

[32] M. Picquart, Lat. Am. J. Phys. Educ. 2, 29 (2008).

[33] X. Ge e N. Er, Interact. Learn. Environ. 13, 139 (2005).

[34] T. Stewart, W. MacIntyre, V. Galea e C. Steel, Interact. Learn. Environ. 15, 77 (2007).

[35] M. Kapur, Cogn. Instr. 26, 379 (2008).

[36] S.T. Bulu e S. Pedersen, Educ. Technol. Res. Dev. 58, 507 (2010).

[37] R.V. Brandão, I.S. Araujo e E.A. Veit, Cad. Bras. Ens. Fís. 28, 507 (2011).

[38] R.V. Brandão, A estratégia da modelagem didáticocientífica reflexiva para a conceitualização do real no Ensino de Física. Tese de Doutorado, Universidade Federal do Rio Grande do Sul, Rio Grande do Sul (2012).

[39] R.V. Brandão, I.S. Araujo e E.A. Veit, Rev. Electr. Invest. Educ. Cienc. 9, 1 (2014).

[40] M.E. Truyol, Z. Gangoso e V.S. López, Lat. - Am. J. Phys. Educ. 6, 1 (2012).

[41] E.S. Ramalho de Souza, Acta Sci. Canoas 14, 309 (2012).
[42] M. Biembengut, Alex. Rev. Educ. Ciênc. Tecnol. 2, 7 (2009).

[43] R.C. Bassanezi, Ensino-aprendizagem com modelagem matemática: uma nova estratégia. (Contexto, São Paulo, 2002).

[44] L.A. Heidemann, I.S. Araujo e E.A. Veit, Cad. Bras. Ens. Fís. 33, 3 (2016).

[45] G. Vergnaud, Investig. Ens. Ciênc. 17, 287 (2012).

[46] X. Ge, L.G. Planas e N. Er, Interdiscip. J. Probl. Learn. 4, 2 (2010).

[47] L.A. Heidemann, I.S. Araujo e E.A. Veit, Rev. Bras. Ens. Fís. 38, 1504 (2016).

[48] C. Ribeiro, Psicol. Reflexão e Crítica 16, 109 (2003).

[49] S. Oh e D.H. Jonassen, Journal of Computer Assisted Learning 23, 95 (2007).

[50] T.I. Pasqualetto, E.A. Veit e I.S. Araujo, Rev. Bras. Pesq. Educ. Ciênc. 17, 551 (2017).

[51] F. Hernandez e M. Ventura, A Organização do Currículo por Projetos de Trabalho: o conhecimento é um caleidoscópio (Artmed, Porto Alegre, 1998).

[52] W.N. Bender, Aprendizagem baseada em projetos: A educação diferenciada para o século XXI (Penso, Porto Alegre, 2015). 